\title{
Increase and regulation of synovial calcitonin gene-related peptide expression in patients with painful knee osteoarthritis
}

This article was published in the following Dove Press journal: Journal of Pain Research

10 May 2017

Number of times this article has been viewed

\author{
Shotaro Takano* \\ Kentaro Uchida* \\ Gen Inoue \\ Atsushi Minatani \\ Masayuki Miyagi \\ Jun Aikawa \\ Dai Iwase \\ Kenji Onuma \\ Manabu Mukai \\ Masashi Takaso
}

Department of Orthopedic Surgery, Kitasato University School of Medicine, Sagamihara, Japan

*These authors contributed equally to this work
Correspondence: Kentaro Uchida Department of Orthopedic Surgery, Kitasato University School of Medicine, I-I5-I Minami-ku, Kitasato, Sagamihara, Kanagawa 252-0374, Japan

$\mathrm{Tel} / \mathrm{Fax}+81427789217$

Email kuchida@med.kitasato-u.ac.jp
Background: Recent studies suggest that the vasodilatory neuropeptide calcitonin gene-related peptide (CGRP) is localized in the synovial tissue and may be involved in the pathology of hip and knee osteoarthritis (OA). However, the regulation and relationship between pain and CGRP expression levels in the synovial tissue of human OA patients are not fully understood.

Methods: Synovial tissues were harvested from 74 participants with radiographic knee OA (unilateral Kellgren/Lawrence grades 3-4) during total knee arthroplasty. CGRP-expressing cells in the resected tissue were identified by immunohistochemical analyses. To examine CGRP expression levels, CD14-positive (CD14+) (macrophage-rich cell fraction) and CD14-negative (CD14-; fibroblast-rich cell fraction) cells were isolated from the synovial tissue. To investigate the involvement of prostaglandin E2 (PGE2) in the regulation of CGRP expression, cultured CD14- and CD14+ cells were stimulated with PGE2. In addition, CGRP expression levels in the synovial tissue of OA patients with strong/severe (visual analog scale [VAS] $\geq 6$ ) and mild/ moderate pain $(\mathrm{VAS}<6)$ were compared.

Results: CGRP-positive cells were detected in the intimal lining layer and comprised both CD14- and CD14+ cells. CGRP expression in non-cultured CD14- fractions was significantly higher than that in CD14+ fractions. The expression levels of CGRP were significantly increased in cultured CD14- cell fractions treated with exogenous PGE2, compared to untreated CD14cell fractions. In contrast, treatment with PGE2 did not increase CGRP regardless of whether or not CD14+ cells expressed CGRP. Furthermore, CGRP expression in the VAS $\geq 6$ group was also significantly higher than that in the VAS $<6$ group.

Conclusion: These findings suggest that CGRP expression in the synovial fibroblasts is regulated by the COX-2/PGE2 pathway and that elevation of synovial CGRP levels may contribute to OA pain.

Keywords: calcitonin gene-related peptide, knee osteoarthritis, regulation, synovium, pain

\section{Introduction}

Patients with knee osteoarthritis (OA) typically present with joint pain, which is typically managed with nonsteroidal anti-inflammatory drugs and opioid analgesics. ${ }^{1}$ However, as these medications are associated with gastrointestinal and cardiorenal toxicity ${ }^{2,3}$ and are ineffective in certain patients, the development of specific and efficacious drugs for OA pain management represents an unmet clinical need.

Calcitonin gene-related peptide (CGRP) is a 37-amino-acid vasodilatory neuropeptide that binds to the calcitonin receptor-like receptor (CLR) and receptor activitymodifying protein 1 (RAMP1). ${ }^{4}$ Recent evidence suggests that CGRP is localized to the synovial tissue and is involved in the pathology of hip and knee arthritis. 5,6 
Specifically, CGRP was detected in the synovial lining layer of patients with developmental dysplasia of the hip, and it stimulated inflammatory cytokine expression in cultured synoviocytes. ${ }^{5}$ CGRP expression in synoviocytes from OA patients was increased by prostaglandin E2 (PGE2). ${ }^{6}$ In addition to fibroblast -like synoviocytes, the synovial lining layer also contains macrophage-like cells, in which levels of CGRP expression have not been determined.

An association between OA pain and CGRP protein levels in several joint tissues from humans has been reported. ${ }^{7,8}$ Notably, CGRP-positive (CGRP+) nerve fibers are increased in the outer

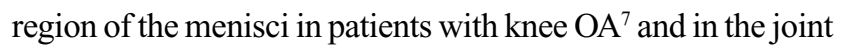
capsule of those with painful hip OA. ${ }^{8}$ Moreover, CGRP expression is also increased in the synovial tissue of OA patients, ${ }^{6,9}$ however, the relationship between pain and CGRP expression in synovial tissue is not well understood in human OA.

Here, we investigated the localization and regulatory mechanisms of CGRP in the knee synovial tissue of patients with different degrees of OA pain.

\section{Materials and methods Patients}

A total of 74 participants with radiographic knee OA (unilateral Kellgren/Lawrence grades 3 [28/74, 38\%] and 4 [46/74, $62 \%]$ ) underwent total knee arthroplasty at our institution. The study population comprised 15 men and 59 women aged 50-88 (mean \pm standard error [SE], 73.9 \pm 0.9 ) years with a mean \pm SE body mass index (BMI) of $26.2 \pm 0.5 \mathrm{~kg} / \mathrm{m}^{2}$ (range $18.2-36.7) \mathrm{kg} / \mathrm{m}^{2}$. Samples of synovial tissue were harvested from each operated knee during the total knee arthroplasty. A portion of each synovial tissue sample was immediately frozen in liquid nitrogen for storage at $-80^{\circ} \mathrm{C}$ until used for RNA extraction. The remaining tissue was fixed for $48 \mathrm{~h}$ in $4 \%$ paraformaldehyde formalin, and tissues from 10 patients were used for histological analysis. In addition, synovial tissues collected from 10 patients were used to isolate CD14+ and CD14cells. The patients were separated into strong/severe (visual analog scale $[\mathrm{VAS}] \geq 6)$ and mild/moderate pain $(\mathrm{VAS}<6)$ groups (Table 1). The use of VAS $=6$ to divide the two groups was based on the classification used in previous studies. ${ }^{10,11}$

This study was approved by the Ethics Review Board of Kitasato University (reference number: KMEO B13-113). All participants of this study provided consent on the day before surgery for the harvesting and use of their synovial tissue.

\section{Immunohistochemistry}

To determine the localization of CGRP, paraformaldehydefixed synovial tissue samples were embedded in paraffin and
Table I Clinical characteristics of patients (VAS $<6$ and $\mathrm{VAS} \geq 6$ groups)

\begin{tabular}{lll}
\hline Characteristics & $\begin{array}{l}\text { VAS }<6 \\
(\mathbf{n}=\mathbf{3 0})\end{array}$ & $\begin{array}{l}\text { VAS } \geq 6 \\
(\mathbf{n}=\mathbf{4 4})\end{array}$ \\
\hline Age (years), mean \pm standard error & $75.5 \pm 1.2$ & $72.8 \pm 1.3$ \\
Male/female $(\mathrm{n})$ & $9 / 21$ & $6 / 38$ \\
BMI $\left(\mathrm{kg} / \mathrm{m}^{2}\right)$, mean \pm standard error & $25.7 \pm 0.7$ & $26.4 \pm 0.7$ \\
Kellgren/Lawrence grades 3/4 patients $(\mathrm{n})$ & $10 / 20$ & $17 / 27$ \\
VAS $(\mathrm{cm})$, mean \pm standard error & $3.6 \pm 0.3$ & $8.4 \pm 0.2$ \\
\hline
\end{tabular}

Abbreviations: VAS, visual analog scale; BMI, body mass index.

sliced into $3-\mu \mathrm{m}$ thick sections. The sections were deparaffinized with xylene for $1 \mathrm{~h}$, hydrated in serial dilutions of ethanol $(100 \%, 95 \%$, and $70 \%)$ and then rinsed in distilled water. The sections were then treated for $6 \mathrm{~h}$ at $4^{\circ} \mathrm{C}$ with rabbit polyclonal primary antibody against CD14 (Abcam, Cambridge, UK) and mouse monoclonal primary antibody against CGRP (Abcam). The sections were further incubated for $1 \mathrm{~h}$ at room temperature with the secondary antibodies Alexa Fluor 488 goat anti-rabbit IgG and Alexa Fluor 594 goat anti-mouse IgG (Thermo Fisher Scientific, Waltham, MA, USA).

\section{Real-time (RT)-PCR analysis}

Total RNA extraction from 74 synovial tissue and cDNA synthesis were performed as described previously. ${ }^{6}$ The sequences of the PCR primer pairs used for RT-PCR analysis are listed in Table 2 . The specificity of the amplified products was examined by melt curve analysis. Quantitative PCR was performed using a Real-Time PCR Detection System (CFX-96; Bio-Rad, Hercules, CA, USA) to determine the relative mRNA expression levels of CGRP in strong/severe (VAS $\geq 6 ; n=30$ ) and mild/moderate pain (VAS $<6 ; n=44$ ) groups. CGRP mRNA expression was normalized to the levels of GAPDH mRNA.

\section{Isolation of CDI4+ and CDI4- cells}

To investigate the involvement of PGE2 in the regulation of CGRP expression, synovial fibroblasts and macrophages were harvested from the synovial tissue collected from the knees of 10 OA patients. The synovial tissue was digested with $40 \mathrm{~mL}$ of $0.1 \%$ type I collagenase to release mononuclear cells, which were then suspended in $500 \mu \mathrm{L}$ phosphate-buffered saline (PBS) containing biotinylated anti-CD14 antibody (Biolegend, San Diego, CA, USA). After incubation for $30 \mathrm{~min}$ at $4^{\circ} \mathrm{C}$, the cells were washed once with PBS, mixed with streptavidin-labeled magnetic particles (BD IMag Streptavidin Particles Plus-DM; BD Biosciences, San Jose, CA, USA), and subjected to magnetic separation using an IMag 
Table 2 Sequences of the primers used in this study

\begin{tabular}{lll}
\hline Primer & Sequence $\left(\mathbf{5}^{\prime} \mathbf{- 3}^{\prime}\right.$ ) & $\begin{array}{l}\text { Product } \\
\text { size (bp) }\end{array}$ \\
\hline CGRP-F & TTGCCCAGAAGAGAGCCTGTG & 91 \\
CGRP-R & TTGTTCTTCACCACACCCCCTG & \\
CDI4-F & TCCCTCAATCTGTCGTTCGC & 150 \\
CDI4-R & ATTCCCGTCCAGTGTCAGGT & \\
GAPDH-F & TGTTGCCATCAATGACCCCTT & 202 \\
GAPDH-R & CTCCACGACGTACTCAGCG & \\
\hline
\end{tabular}

Abbreviations: CGRP, calcitonin gene-related peptide; $F$, forward; $R$, reverse.

separation system (BD Biosciences) to isolate labeled cells. After an 8-min incubation at room temperature, unbound (CD14-) cells were collected by the addition of warmed $\left(37^{\circ} \mathrm{C}\right) \alpha$-minimum essential culture medium $(\alpha$-MEM) to the cell suspension. The tube containing bound cells was removed from the magnetic support, and CD14+ cells were collected by the addition of $3 \mathrm{~mL} \alpha$-MEM. The CD14+ and CD14- cell fractions were centrifuged at $300 \mathrm{~g}$ for $10 \mathrm{~min}$. CD14+ and CD14- cell fractions from five patients were directly used for quantitative PCR analysis of CGRP, whereas those from the other five patients were cultured in six-well plates containing $\alpha$-MEM. After 7 days of culture, the cells were stimulated with PGE2 $(10 \mu \mathrm{M})$ for $24 \mathrm{~h} . \alpha$-MEM containing no PGE2 was added to vehicle control cells and incubated for $24 \mathrm{~h}$. The treated and control cells were harvested for RNA isolation and CGRP expression, which was performed by RT-PCR analysis.

\section{Statistical analysis}

The Mann-Whitney $U$ test was used to compare the differences between CD14- and CD14+ cells, vehicle control and PGE2-treated cells, and CGRP expression in the strong/ severe (VAS $\geq 6$ ) and mild/moderate pain (VAS $<6$ ) groups. A $p$-value of $<0.05$ was considered statistically significant for all analyses. All statistical analyses were performed using SPSS software (v. 19.0; SPSS, Chicago, IL, USA).

\section{Results}

\section{Localization and expression of CGRP in synovial tissue of OA patients}

To determine the cell type(s) that expresses CGRP in the synovial lining layer of knees in OA patients, immunohistochemical analysis of CGRP and CD14 was performed (Figure 1A-E). CD14+ cells (macrophages) were localized to the intimal lining layer (Figure 1B). Furthermore, CGRP+ cells were also observed in the intimal lining layer and corresponded to both CD14+ and CD14- cells (fibroblasts; Figure 1C-E). However, RT-PCR analysis revealed that CGRP expression in CD14- fractions was significantly higher than that in CD14+ fractions (Figure 1F, $p=0.020$ )

\section{Effects of PGE2 on CGRP expression} in cultured synovial fibroblasts and macrophages

CGRP expression in cultured cells isolated from synovial tissue was measured after treatment with or without exogenous PGE2. CD14 mRNA expression in CD14+ cells was significantly higher than that in CD14- cells (Figure 2A, $p=0.016$ ). CGRP expression increased significantly in CD14cell fractions treated with PGE2, compared to vehicle control CD14- cells (Figure 2B, $p=0.008$ ). In contrast, treatment with PGE2 did not increase CGRP regardless of whether or not CD14+ cells expressed CGRP (Figure 2B, $p=1.000$ ).

\section{CGRP expression in the VAS $<6$ and $V A S \geq 6$ groups}

To investigate the relationship between synovial CGRP expression levels and pain, the OA patients were separated into strong/severe $(\mathrm{VAS} \geq 6$ ) and mild/moderate pain $(\mathrm{VAS}<6$ ) groups. There were no differences between the two groups in patient age, male/female ratio, BMI, or KL 3/4 ratio (Table 1). RT-PCR analysis indicated that CGRP expression in the synovial tissue of the VAS $\geq 6$ group was significantly higher than that in the VAS $<6$ group (Figure 3, $p=0.035$ ).

\section{Discussion}

In the synovial tissue of patients with knee OA, CD14- cells in the synovial lining layer highly expressed CGRP as compared to CD14+ cells. The treatment of CD14- cells with PGE2 stimulated CGRP expression. In addition, CGRP expression in the VAS $\geq 6$ group of OA patients was significantly higher than that in the VAS $\geq 6$ group. Taken together, these findings suggest that synovial fibroblast-derived CGRP in the lining layer may be involved in mediating joint pain in $\mathrm{OA}$.

Synovial tissue contains macrophage- and fibroblast-like cells in the lining layer. ${ }^{12-14}$ Previous studies reported that CGRP+ cells are present in the synovial lining layer of developmental dysplasia of the hip ${ }^{5}$ and OA patients; ${ }^{6}$ however, cell types that expressed CGRP were not determined. Here, double immunostaining of synovial tissue for CGRP and the synovial macrophage marker, CD14, revealed that CGRP+ cells corresponded to CD14- and CD14+ cells, although CGRP expression in CD14- cells was significantly higher than in CD14+ cells. This suggests that synovial fibroblasts are one of the main sources of CGRP production in synovial tissues.

CGRP expression is regulated by inflammatory cytokines, growth factors and PGE2 in several cell types, such as immune, ${ }^{15-17}$ epithelial, ${ }^{18}$ neural ${ }^{19,20}$ and synovial cells. ${ }^{6}$ 

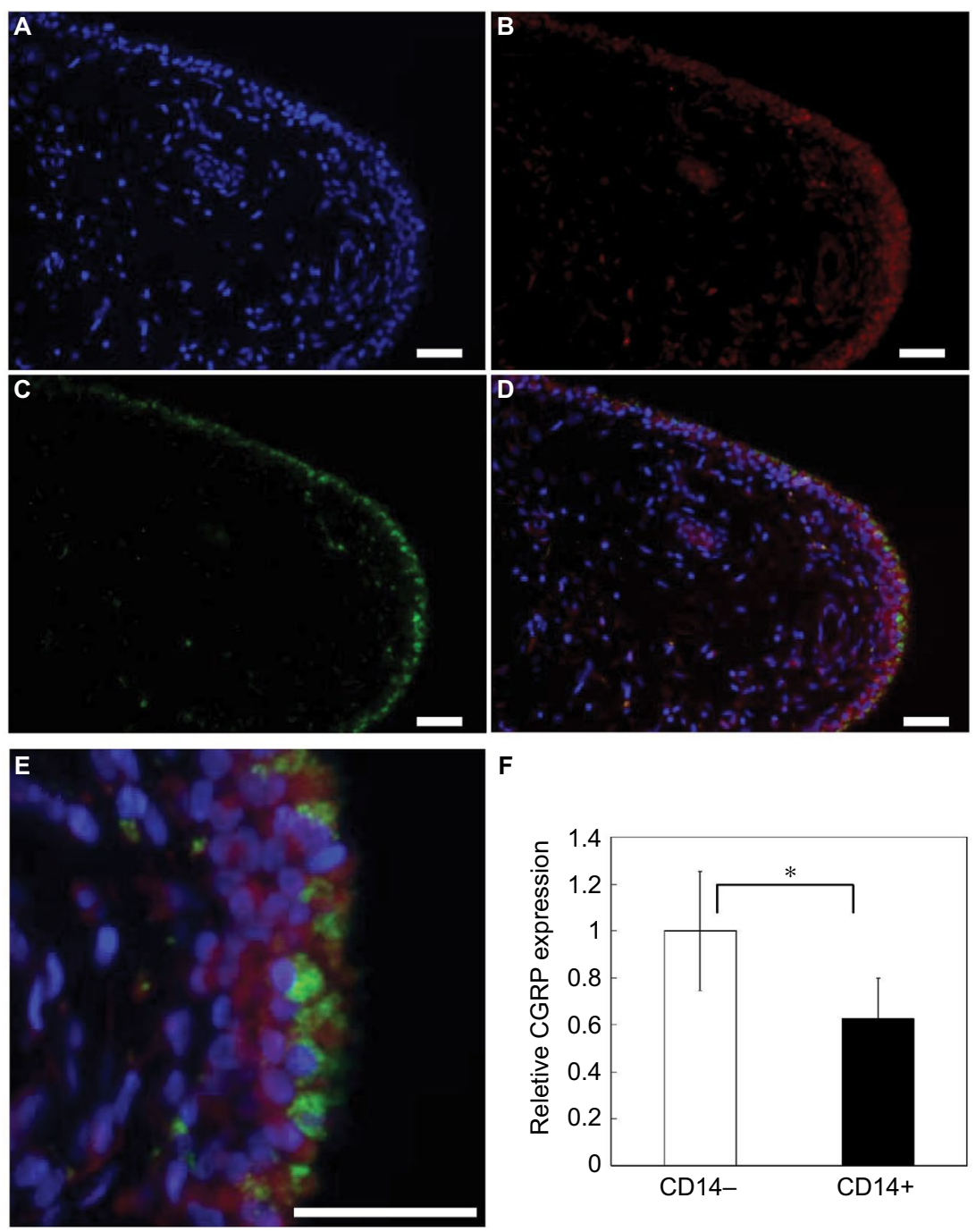

$\mathbf{F}$

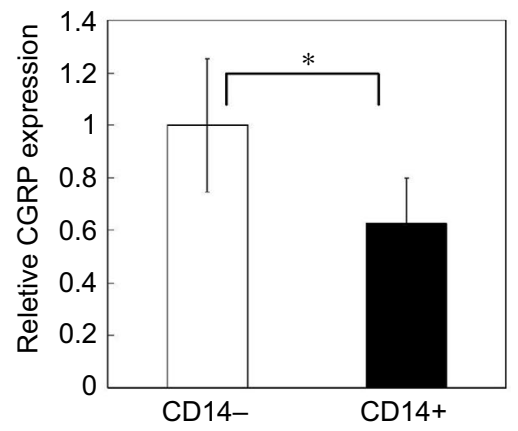

Figure I CGRP immunolocalization and mRNA expression in synovial tissue.

Notes: Synovial tissue stained with (A) DAPI (nuclei), (B) CDI4 or (C) CGRP. (D) Merged image. (E) High-magnification merged image of (D). Scale bars $=100 \mu \mathrm{m}$. (F) Real-time PCR analysis of CGRP expression in non-cultured CDI4- and CDI4+ fractions. All data are presented as mean \pm standard error $(n=5)$. ${ }^{*} p<0.05$. Abbreviations: CGRP, calcitonin gene-related peptide; DAPI, 4',6-diamidino-2-phenylindole; PCR, polymerase chain reaction.

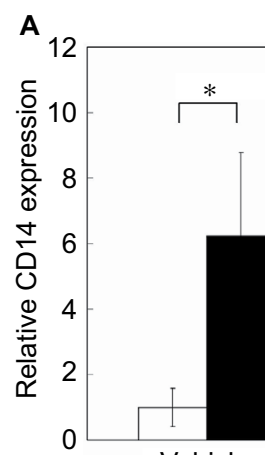

Vehicle

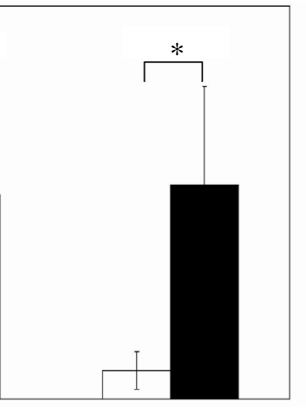

PGE2
B

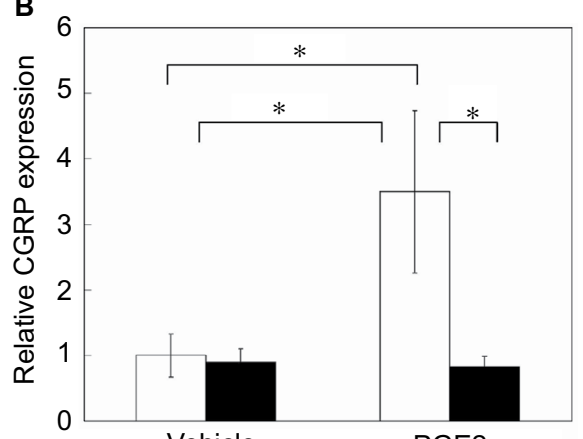

Vehicle
PGE2

$\square$ CD14- $\quad$ CD14+

Figure 2 Effects of PGE2 on CGRP expression in cultured synovial fibroblasts and macrophages.

Notes: Synovial fibroblasts and macrophages were stimulated in vitro with PGE2 (10 $\mu$ M) or without PGE2 (vehicle) for $24 \mathrm{~h}$ prior to the extraction of total RNA for (A) CDI4 and (B) CGRP expression analysis. All data are presented as mean \pm standard error $(n=5)$. * $p<0.05$.

Abbreviations: PGE2, prostaglandin E2; CGRP, calcitonin gene-related peptide. 


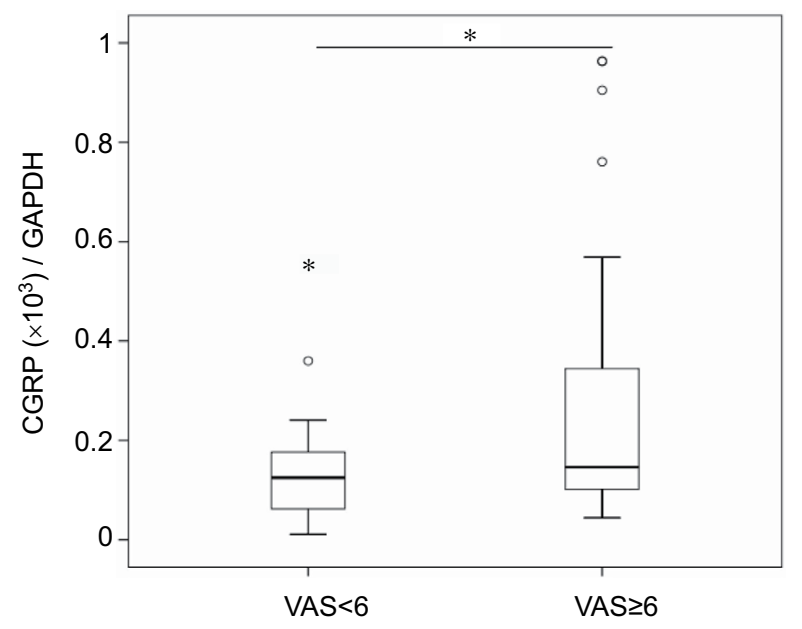

Figure 3 CGRP expression in OA patients with strong/severe (VAS $\geq 6$ ) and mild/ moderate pain $(\mathrm{VAS}<6)$.

Notes: Real-time PCR analysis of CGRP expression in synovial tissue of OA patients. Box and Whisker plot for CGRP expression in synovial tissue of OA patients with strong/severe (VAS $\geq 6 ; n=44$ ) and mild/moderate pain (VAS $<6 ; n=30$ ). The plot shows median (bold horizontal line), IQR (box) and range, with outliers plotted beyond I.5 times the IQR from the box. ${ }^{*} p<0.05$.

Abbreviations: CGRP, calcitonin gene-related peptide; OA, osteoarthritis; VAS, visual analog scale; PCR, polymerase chain reaction; IQR, interquartile range.

In a previous study, a correlation between CGRP and COX-2 expression was detected in the synovial tissue of 43 patients, and the enzymatic product of COX-2 (PGE2) also stimulated CGRP gene expression in synovial cell cultures containing fibroblasts and macrophages. ${ }^{6}$ Here, PGE2 stimulated CGRP expression in isolated CD14-(fibroblast-rich fraction) cells, but not in CD14+ (macrophage-rich fraction) cells. Taken together, these results suggest that CGRP expression in synovial fibroblasts, but not in macrophages, is regulated by the COX-2/PGE2 pathway.

Several studies on the joint tissue of OA patients have suggested that the elevation of CGRP levels is associated with OA pain. ${ }^{7,8}$ For example, CGRP-expressing nerve fibers are increased in the outer region of the menisci of patients with knee OA. ${ }^{7}$ The density of CGRP-expressing nerve fibers in the joint capsule of patients with hip OA and severe pain was also higher than that found in asymptomatic controls. ${ }^{8}$ In addition, patients with a painless failed hip arthroplasty had no CGRP-expressing fibers surrounding the joint. ${ }^{8}$ Here, OA patients with severe/strong pain had increased synovial CGRP expression levels. Based on these findings, synovial CGRP appears to play an important role in the mechanism of pain production in OA patients.

Basic studies have demonstrated that CGRP and CGRP receptor antagonists are efficacious for OA pain. ${ }^{21-25}$ In monosodium iodoacetate (MIA) and medial meniscus OA models in rats, treatment with a CGRP receptor antagonist normalized the enhanced mechanical-evoked responses of joint nociceptors. ${ }^{22}$ Furthermore, the small-molecule CGRP receptor antagonist BIBN4096BS also exhibited analgesic effects in a rat MIA model. ${ }^{23}$ In addition, humanized antibody against CGRP relieved pain in a rat MIA model, ${ }^{21,24}$ and clinical trials of an anti-CGRP antibody in human OA patients are currently underway. ${ }^{25}$ The findings from the present study may provide valuable information for developing more effective pain treatments for OA.

Several limitations of the present study warrant mention. First, the lack of inclusion of a control, non-OA patient population decreases the certainty of these results. Future experiments are needed to confirm whether CGRP levels are elevated in the synovial tissues of OA patients as compared to non-OA patients. Second, although the present data provide evidence that altered synovial CGRP levels are associated with OA pain, it remains to be determined whether a direct causative link exists between CGRP. Finally, the concentration of CGRP protein in synovial tissue was not determined.

\section{Conclusion}

Synovial fibroblasts are shown to be one of the main sources of CGRP in synovial tissues, and the expression of CGRP appears to be regulated by the COX-2/PGE2 pathway. Elevated synovial CGRP expression was associated with increased pain in OA patients with severe/strong pain. The findings from the present study suggest that modifying the regulation of CGRP expression in synovial tissue may be a promising therapeutic strategy for managing OA pain.

\section{Acknowledgments}

The authors thank Ms. Yuko Onuki for her assistance with the immunohistochemistry analysis. This investigation was supported in part by JSPS KAKENHI grant number 15K20016, the Uehara Memorial Foundation, a Kitasato University Research Grant for Young Researchers, and research grants from the Parents' Association of Kitasato University School of Medicine.

\section{Disclosure}

The authors report no conflicts of interest regarding this work.

\section{References}

1. Zhang W, Moskowitz RW, Nuki G, et al. OARSI recommendations for the management of hip and knee osteoarthritis, part II: OARSI evidence-based, expert consensus guidelines. Osteoarthritis Cartilage. 2008;16(2):137-162.

2. Johnsen SP, Larsson H, Tarone RE, et al. Risk of hospitalization for myocardial infarction among users of rofecoxib, celecoxib, and other NSAIDs: a population-based case-control study. Arch Intern Med. 2005;165(9):978-984.

3. Whelton A. Renal and related cardiovascular effects of conventional and COX-2-specific NSAIDs and non-NSAID analgesics. Am J Ther. 2000;7(2):63-74. 
4. Walker CS, Conner AC, Poyner DR, Hay DL. Regulation of signal transduction by calcitonin gene-related peptide receptors. Trends Pharmacol Sci. 2010;31(10):476-483.

5. Wang H, Zhang X, He JY, et al. Increasing expression of substance $P$ and calcitonin gene-related peptide in synovial tissue and fluid contribute to the progress of arthritis in developmental dysplasia of the hip. Arthritis Res Ther. 2015;17:4.

6. Minatani A, Uchida K, Inoue G, et al. Activation of calcitonin generelated peptide signaling through the prostaglandin E2-EP1/EP2/EP4 receptor pathway in synovium of knee osteoarthritis patients. J Orthop Surg Res. 2016;11(1):117.

7. Ashraf S, Wibberley H, Mapp PI, Hill R, Wilson D, Walsh DA. Increased vascular penetration and nerve growth in the meniscus: a potential source of pain in osteoarthritis. Ann Rheum Dis. 2011;70(3):523-529.

8. Saxler G, Loer F, Skumavc M, Pfortner J, Hanesch U. Localization of SP- and CGRP-immunopositive nerve fibers in the hip joint of patients with painful osteoarthritis and of patients with painless failed total hip arthroplasties. Eur J Pain. 2007;11(1):67-74.

9. Saito T, Koshino T. Distribution of neuropeptides in synovium of the knee with osteoarthritis. Clin Orthop Relat Res. 2000;(376):172-182.

10. Arendt-Nielsen L, Nie H, Laursen MB, et al. Sensitization in patients with painful knee osteoarthritis. Pain. 2010;149(3):573-581.

11. Collins SL, Moore RA, McQuay HJ. The visual analogue pain intensity scale: what is moderate pain in millimetres? Pain. 1997;72(1-2):95-97.

12. Barland P, Novikoff AB, Hamerman D. Electron microscopy of the human synovial membrane. J Cell Biol. 1962;14:207-220.

13. Graabaek PM. Characteristics of the two types of synoviocytes in rat synovial membrane. An ultrastructural study. Lab Invest. 1984;50(6):690-702.

14. Mucke J, Hoyer A, Brinks R, et al. Inhomogeneity of immune cell composition in the synovial sublining: linear mixed modelling indicates differences in distribution and spatial decline of CD68+ macrophages in osteoarthritis and rheumatoid arthritis. Arthritis Res Ther. 2016;18:170.

15. Bracci-Laudiero L, Aloe L, Buanne P, et al. NGF modulates CGRP synthesis in human B-lymphocytes: a possible anti-inflammatory action of NGF? J Neuroimmunol. 2002;123(1-2):58-65.
16. Bracci-Laudiero L, Aloe L, Caroleo MC, et al. Endogenous NGF regulates CGRP expression in human monocytes, and affects HLADR and CD86 expression and IL-10 production. Blood. 2005;106(10): 3507-3514.

17. Ma W, Dumont Y, Vercauteren F, Quirion R. Lipopolysaccharide induces calcitonin gene-related peptide in the RAW264.7 macrophage cell line. Immunology. 2010;130(3):399-409.

18. Li W, Hou L, Hua Z, Wang X. Interleukin-1beta induces beta-calcitonin gene-related peptide secretion in human type II alveolar epithelial cells. FASEB J. 2004;18(13):1603-1605.

19. Bowen EJ, Schmidt TW, Firm CS, Russo AF, Durham PL. Tumor necrosis factor-alpha stimulation of calcitonin gene-related peptide expression and secretion from rat trigeminal ganglion neurons. J Neurochem. 2006;96(1):65-77.

20. Neeb L, Hellen P, Boehnke C, et al. IL-1beta stimulates COX-2 dependent PGE(2) synthesis and CGRP release in rat trigeminal ganglia cells. PLoS One. 2011;6(3):e17360.

21. Benschop RJ, Collins EC, Darling RJ, et al. Development of a novel antibody to calcitonin gene-related peptide for the treatment of osteoarthritis-related pain. Osteoarthritis Cartilage. 2014;22(4):578-585.

22. Bullock CM, Wookey P, Bennett A, Mobasheri A, Dickerson I, Kelly S. Peripheral calcitonin gene-related peptide receptor activation and mechanical sensitization of the joint in rat models of osteoarthritis pain. Arthritis Rheumatol. 2014;66(8):2188-2200.

23. Hirsch S, Corradini L, Just S, Arndt K, Doods H. The CGRP receptor antagonist BIBN4096BS peripherally alleviates inflammatory pain in rats. Pain. 2013;154(5):700-707.

24. Puttfarcken PS, Han P, Joshi SK, et al. A-995662 [(R)-8-(4-methyl-5(4-(trifluoromethyl)phenyl)oxazol-2-ylamino)-1,2,3,4-tetrahydronaphthalen-2-ol], a novel, selective TRPV1 receptor antagonist, reduces spinal release of glutamate and CGRP in a rat knee joint pain model. Pain. 2010;150(2):319-326.

25. Walsh DA, Mapp PI, Kelly S. Calcitonin gene-related peptide in the joint: contributions to pain and inflammation. Br J Clin Pharmacol. 2015; 80(5):965-978.
Journal of Pain Research

\section{Publish your work in this journal}

The Journal of Pain Research is an international, peer reviewed, open access, online journal that welcomes laboratory and clinical findings in the fields of pain research and the prevention and management of pain. Original research, reviews, symposium reports, hypothesis formation and commentaries are all considered for publication.

\section{Dovepress}

The manuscript management system is completely online and includes a very quick and fair peer-review system, which is all easy to use. Visit http://www.dovepress.com/testimonials.php to read real quotes from published authors. 